\title{
La educación en valores hoy en día: entre conciencia crítica y respuestas constructivas
}

\author{
Amalia Bernardini
}

Profesora Investigadora.UNED; abernardcr@yahoo.com

Recibido: 4 de mayo de 2010

\section{RESUMEN}

Frente a la crisis ética y moral, es aún válido apelar a la educación en valores, que, sin embargo, se enfrenta al reto de la relativización de los mismos, tanto práctica, como teórica. Pero, el relativismo y la separación de la ética de las diferentes actividades humanas como la política, la tecno-ciencia y la economía, es disociadora para la sociedad y destructiva para la vida. Por otro lado, el absolutismo valorativo puede conducir a dogmatismo, intolerancia y autoritarismo. La construcción de un universalismo axiológico no dogmático es un problema abierto, pero cada vez más sensible. Las modalidades educativas actuales son múltiples, pero, entre ellas, serán más relevantes las que favorezcan una formación moral $y$, en general, en valores, aparejada con la formación de la inteligencia; vinculada con la integralidad de la persona; transversal al curriculum y que eduque en valores por medio de valores presentes y tangibles, convertidos en experiencia.

\section{PALABRAS CLAVE}

Educación, filosofía, pedagogía, valores, axiología, ética, moral.
Aceptado: 24 de setiembre de 2010

\section{KEY WORDS}

Education, philosophy, pedagogy, values, axiology, ethics, moral.

\section{- Valores en crisis y educación}

Que la sociedad contemporánea sufre una crisis de valores es un tópico y hasta un decir; que ha perdido muchos de los que se tenían y que necesita adquirir o remozar otros valores es obvio. Se ha dicho, con razón, que no se han perdido los valores, sino la congruencia y la coherencia social acerca de ellos, de modo que no hay una continuidad valorativa (o axiológica) entre las entidades tradicionalmente formadoras: la familia, la escuela, la iglesia, la política, los grupos y centros de recreación y esparcimiento, entre otros. 
Existe lo que Edgar Morin llama "un sentimiento generalizado de asfixia ética" (La Méthode, 2004: 190). Si bien es cierto que los valores en crisis no son sólo los éticos, la ausencia o carencia de éstos, así como también de los valores ciudadanos, es lo que más preocupa, porque ataca a la misma posibilidad de la convivencia social y política, o lo que se ha llamado "el contrato social". Thomas Hobbes decía, a mediados del siglo XVII, que la violencia "la guerra de todos contra todos" hace imposible tanto la convivencia como la vida individual. Hoy podemos atribuir la misma responsabilidad a la corrupción moral y a la falta o no vivencia de valores.

Por otra parte, la sociedad nuestra, la costarricense, es una sociedad que cree en la educación. La prueba es que cuando nos encontramos frente $a$ un problema que tiene que ver con decisiones y conducta humana y social (vicios, corrupción, violencia, salud, prevención de accidentes, maltrato al ambiente, modales irrespetuosos, etc.), inmediatamente nos dirigimos a la educación, que, nos parece, debería ser capaz de prevenir todos los problemas de conducta indeseable o defectuosa. Por eso, sigue siendo válido y oportuno hablar de educación en valores.

El enseñar conductas deseables es relativamente fácil (se llama "adiestrar", a veces, "domesticar"); sin embargo, el proceso educativo no se puede reducir a la inducción de conductas, básicamente porque el ser humano es más que su conducta. Si solo suponemos que es "persona", entonces implicamos la noción de libertad, o al menos, de motivaciones interiores de las actuaciones externas. Tales motivaciones interiores tienen que ver con inteligencia, conciencia, voluntad y emociones. Se trata entonces de que las motivaciones lleguen a ser, en la medida de lo posible, conscientes, autónomas, emocionalmente inteligentes e inspiradas en valores.

\section{- Apuntes sobre Axiología}

Con esto, volvemos al tema: ¿Qué son los valores? ¿Por qué valen los valores? ¿Por qué valen para algunos, para algunas generaciones o épocas? Y, la pregunta clave: ¿son subjetivos u objetivos? Pregunta clave porque la subjetividad parece principio de no universalidad $y$, por lo tanto, de imposibilidad de un acuerdo o consenso sobre los valores. De hecho, como afirma José Ferrater Mora (1958: 1377):

"La tensión entre el extremo relativismo y el absolutismo extremo se ha apaciguado en cierto modo, cuando se ha reconocido, por un lado, que el valor no puede estar sometido a la arbitrariedad subjetiva $y_{1}$ por otro, que el valor carece de sentido si no es referido a una persona que lo estime".

Sin embargo, la subjetividad de los valores sería parecida a la de las formas a priori kantianas, que garantizan la universalidad del conocimiento y del juicio reflexivo, aunque no el contacto con la "cosa en sí". La filosofía antigua y medieval se ocupó del ser y no puso en duda que éste fuera conocible como tal por el sujeto. Entonces, el valor se hallaba íntimamente unido con el Ser. El Ser, en tal perspectiva, era bueno, bello y, en cuanto conocido, verdadero, siendo el mal atribuido al no ser, o a la limitación del ser. En el ámbito ético, se hablaba de virtudes, no de valores $y$, sobre todo en la Edad Media, virtuoso era el que adecuaba sus actos al orden del Ser y a la jerarquía de los seres.

Así, pues, la reflexión autónoma sobre los valores es un producto de finales del siglo XVIII. En la Edad Moderna, sobre todo por obra del Empirismo y de Kant, se sustituyó la meditación antigua y medieval sobre el ser por la meditación sobre el conocimiento, sus condiciones y sus límites. El Racionalismo también inició como discurso metodológico sobre el método y las condiciones del conocimiento, pero no dejó de ser metafísico, o sea, de pronunciarse sobre la realidad en sí (o substancia). Tanto es así que los empiristas y Kant lo acusaron de "dogmático", por pretender pronunciarse sobre la realidad antes de examinar si el conocimiento humano estaba en condiciones para ello. 
De modo que una teoría de los valores hunde sus raíces en el formalismo kantiano. En el contexto de la teoría pura de los valores o axiología pura, entonces, aunque esta trate los valores como "entidades" objetivas o "cualidades irreales", a saber, no corporales, éstos están inevitablemente vinculados con la conciencia o capacidad valorativa del ser humano y, aún cuando se declaren universales y absolutos, siempre lo serán en un contexto cultural o histórico.

El carácter tardo-moderno de la reflexión sobre los Valores puede advertirse con toda claridad en el pensamiento de Federico Nietzsche (1844-1900), quien "interpreta las actitudes filosóficas no como posiciones del pensamiento ante la realidad, sino como actitudes frente a los valores" (Ferrater Mora, 1958: 1376).

Uno de los argumentos fundamentales del pensamiento de Nietzsche era que los valores tradicionales, representados por los valores de la cultura moderna, que él ve encarnados en el cristianismo, el socialismo y el igualitarismo democrático, como: objetividad, bondad, humildad, conformidad, piedad, amor al prójimo, etc., son valores inferiores. Representan, en efecto, una moral de esclavos, de débiles y resentidos, pues la vida y su afirmación, el poder y su afirmación son superiores a ellos y exigen la creación de una nueva estimativa, una "inversión de todos los valores", donde la objetividad es sustituida por la personalidad creadora; la bondad por la virtud, en el sentido del Renacimiento italiano y la "areté" griega; la humildad por el orgullo; la conformidad por el riesgo; la piedad por la crueldad y el amor al prójimo por el amor a lo lejano. La cultura europea, al haber abrazado una falsa tabla de valores, está condenada al hundimiento y en la decadencia. Sólo se salvarán los que sientan como propia la necesidad de superar lo "humano demasiado humano" por el "superhombre".

Ahora bien, desde Sócrates y Aristóteles se había extendido al pensamiento occidental la idea que el ser humano perfecto es el que cumple con la norma. Sin embargo, los "maestros de la sospecha" (llamados así por Paul Ricoeur), Marx, Nietzsche y Freud, se colocan más allá de la ley, relativizándola como expresión de la clase dominante (Marx), o colocándola "más allá del bien y del mal" (Nietzsche) o, finalmente, haciéndola expresión de un super-Ego censor, represor o sublimador de pulsiones elementales (Freud).

Max Scheler (1874-1928), es considerado pensador de gran importancia en el desarrollo de la filosofía de los valores, como también de la antropología filosófica, de la filosofía de la religión y de la sociología del saber. Seguidor de Husserl, se convirtió, junto a Heidegger, en uno de los primeros fenomenólogos que interpretó a su manera el método del maestro Husserl. Scheler utilizó la fenomenología para estudiar los fenómenos emocionales y sus respectivas intencionalidades (los valores). Este autor consideró que la Fenomenología había quedado, con Husserl, injustificadamente limitada a la descripción de la intencionalidad intelectual o (en el último Husserl) a la exploración de la conciencia pura. Los valores son esencias puras, intemporales y absolutamente válidas como las esencias husserlianas, pero, de ellas, no puede predicarse ni la inteligibilidad racional, ni el carácter lógico. Los valores, para Scheler, poseen intencionalidad emocional y están sometidos a leyes que no son ni inferiores ni superiores a las lógicas, sino sencillamente distintas.

Scheler distinguió las esencias de aquello que era tangible, real y existente, cosa que lo llevó a la afirmación de la independencia de los valores, eternos e inmortales, de los bienes, que serían sus portadores sólo circunstanciales.

A diferencia del formalismo moral kantiano, Scheler afirma que su punto de vista ético es un "apriorismo moral material", lo cual significa que los valores son, en cuanto esencias puras, elementos a priori y también elementos materiales. Los resultados de esta síntesis de lo material con lo a-priori en el campo emocional conducen a Scheler a un sistema de valores articulados jerárquicamente, en donde se supera el relativismo de toda ética material, por la esencial 
aprioridad de los valores. Además, en su famosa obra El formalismo en la ética y la ética material de los valores, trata de dar un nuevo fundamento personalista a la ética, ya que los valores se presentan de modo directo inmediato a la persona, y no simplemente a la conciencia.

José Ortega y Gasset (1883-1955), en su ensayo Introducción a una Estimativa ¿Qué son los valores?, siguió la senda trazada por Scheler. La Estimativa es, precisamente, la teoría de los valores, llamada también Axiología, a saber, teoría de lo que es "axios", digno o estimable.

Max Scheler insistió en separar el orden del "ser" del orden del "valer" para poner de relieve que la aproximación racional cognoscitiva es insuficiente para captar la esencia del valor. Los objetos que "son" pueden ser comprendidos en sí mismos y no necesariamente en "polaridades", como los valores.

Max Scheler, Ortega y Gasset y otros autores han llegado a atribuirles a los valores características ya bien conocidas para quien se ha acercado al tema de la axiología; casi podríamos decir que hay una escolástica axiológica, porque, a diferencia de casi todos los campos de la filosofía, hay pautas bastante compartidas por los autores que se han ocupado del tema:

A. El valer. Hay un grupo de objetos que no puede caracterizarse por el ser, como los objetos reales y los ideales. De estos objetos se dice, según la expresión del filósofo Rudolph Hermann Lotze, que valen y que, por lo tanto, no tienen ser, sino valer. Los objetos reales tienen especialidad, temporalidad, causalidad, etc. Los objetos ideales son intemporales, como los valores, cuya forma de ser no es, sin embargo, ni el ser ideal ni el real, sino el ser valioso. Luis Lavelle dijo que en esta definición del valor se supone una conciencia, que aprueba, o no, algo.

B. Objetividad. Los valores son objetivos, es decir, no dependen de las preferencias individuales (véase Scheler y Ortega, inspirado en él). Sin embargo, la teoría relativista de los valores (véase los austriacos von Meinong y Ehrenfels) sostiene que los actos de agrado o desagrado, así como nuestro deseo son el fundamento de los valores. La teoría relativista sostiene que posee valor lo deseable; la absolutista en cambio, que es deseable lo valioso. Los relativistas desconocen la forma peculiar e irreductible de realidad de los valores, en tanto que los absolutistas a veces desconocen los problemas planteados por la relación efectiva entre los valores y la realidad humana e histórica. Los valores no son entidades trascendentes. La objetividad del valor sólo significa su autonomía con respecto a toda estimación subjetiva o arbitraria. Por ejemplo, el mismo Ortega, quien afirma que los valores son objetivos, tanto es así que habla de una "ceguera hacia los valores" en ciertas circunstancias o épocas históricas, reconoce en cierto sentido su subjetividad al afirmar (en sentido parecido a Lavelle) que los valores existen sólo para sujetos dotados de facultad estimativa.

C. No independencia. Los valores no son independientes ontológicamente, sino que necesariamente son adheridos a las cosas. Los valores hacen siempre referencia al ser y son expresados como predicaciones del ser.

D. Polaridad. Los valores se presentan siempre en un aspecto positivo y uno negativo, porque no son entidades indiferentes como las otras realidades. Así, a la belleza se contrapone la fealdad; a la bondad, la maldad; a la justicia, la injusticia, etc. El aspecto negativo del valor se llama también disvalor o contra-valor.

E. Cualidad. Los valores son totalmente independientes de la cantidad y por eso no pueden establecerse relaciones cuantitativas entre las cosas valiosas. 
Lo característico de los valores es la cualidad pura.

F. Jerarquía. Es común hablar de los valores en una tabla en que están ordenados jerárquicamente. Esta caracterización de los valores corresponde a la axiología formal, mientras que la axiología material estudia más concretamente las cuestiones que afectan la relación entre los valores y la vida humana. Aquí también hay influencia de las perspectivas subjetivistas y objetivistas. En el primer caso, los valores se hallan en la vida humana y son determinados, en su ser y su jerarquía, por ella. En el segundo caso, los valores son simplemente descubiertos por el hombre. La concepción objetivista de los valores admite la posibilidad de una ceguera para el valor o para ciertos valores en ciertas formas de vida o épocas históricas. De modo que el conocimiento y aprecio de los valores es limitado y puede aumentar con nuevas perspectivas sobre los valores en el curso del acontecer histórico. Por ejemplo, según Max Scheler, los valores se dividen, de menor a mayor, en:

- Valores de lo agradable, ejemplo: dulce y amargo, según me agrade.

- Valores vitales, que se refieren a lo físico, ej. sano y enfermo.

- Valores espirituales, que se dividen en tres tipos:

- Estéticos, ej. bello y feo

- Jurídicos, referentes a lo social, p. ej. justo e injusto

- Intelectuales, los del conocimiento

- Valores religiosos, p. ej. sacro y profano

Ortega y Gasset formula una clasificación similar:

- Valores de lo útil, que hacen referencia a algo de alguna cosa, por ej. capaz-incapaz, caro-barato, abundante-escaso.
- Valores vitales, que hacen referencia a la persona, por ej. sano-enfermo, selecto-vulgar, enérgico-inerte, fuerte-débil.

- Valores espirituales, que se dividen en tres tipos:

- Intelectuales, referentes al conocimiento, por ej. conocimiento-error, exactoaproximado, evidente-probable.

- Morales, referentes a lo social, por ejemplo: bueno-malo, bondadosomalvado, justo-injusto, escrupulosorelajado, leal-desleal.

- Estéticos, referentes a la belleza, por ej. bello-feo, gracioso-tosco, eleganteinelegante, armonioso-inarmónico.

- Valores religiosos, con referencia a Dios: santo (o sagrado)-profano, divino-demoníaco.

Uno de los problemas más difíciles de la axiología material es el de las relaciones entre valores y concepción del mundo, porque, como dice Ferrater Mora "su solución depende a su vez en parte considerable de la concepción del mundo vigente o sustentada por el investigador" (p.1379). Por otra parte, este problema parece agudizarse hoy en día y al mismo tiempo tender hacia una visión menos dependiente de las concepciones del mundo, porque estamos en búsqueda de principios éticos universales, o sea, aceptables por toda la humanidad, más allá, precisamente, de las creencias, las doctrinas y las visiones de mundo particulares.

\section{- La historia de la educación y nosotros}

Toda la historia de la educación, que prácticamente coincide con la de la humanidad, abunda en ejemplos de comportamientos humanos deseados por la sociedad e inducidos con métodos más o menos autoritarios. Tipos humanos bien definidos en las diferentes 
épocas históricas: el guerrero, el sacerdote, el caballero andante, el monje, y tipos humanos más ligados a los orígenes y desarrollo de la modernidad: el artesano luterano, el religioso jesuita, el caballero inglés, el diplomático decimonónico, el soldado y el oficial de los ejércitos modernos, todos formados con enorme eficacia, siguiendo criterios y métodos establecidos intuitivamente, o sea, sin preocupación por fundamentar científicamente los métodos de enseñanza y con base en valores establecidos por la sociedad, y no discutidos, aunque, muchas veces muy respetables, como la valentía, la lealtad, la generosidad, la piedad religiosa, la laboriosidad, etc.

¿Por qué a nosotros nos cuesta tanto formar eficazmente a nuestra juventud en valores humanos e intelectuales? Debe ser que la sociedad, la familia, el sistema educativo o nosotros mismos, como padres y educadores, carecen o carecemos de los valores que se pretenden formar. Debe ser, además, que no tenemos claro en nuestra mente cuáles valores enseñar y por qué son deseables. Es precisamente un síntoma de la crisis de valores de nuestra sociedad la inseguridad que tenemos como educadores sobre cuáles valores proponer y por qué. Por ejemplo: ¿Educar para la socialización o para el individualismo? ¿Para la solidaridad o para la competencia? ¿Qué sentido tiene educar para la integridad, si el joven se debe enfrentar constantemente a la corrupción? O, ¿Tiene sentido inspirar admiración para la perfección y la excelencia, cuando está tan difundida, y exitosa, la mediocridad?

Estamos enfrentados a la posibilidad de un completo relativismo, que, desde el punto de vista ético y de la convivencia humana, es disociador.

Si luego echamos una mirada planetaria, vemos que, en nuestro mundo, hay diferencias radicales en el aprecio hacia valores: lo que en una parte es un anti-valor como el irrespeto de los derechos humanos, es piedad religiosa en otro lado; lo que para nosotros es locura homicida y suicida es heroísmo en otro lado y así sucesivamente.

Por otra parte, de este carácter disociador del relativismo estamos tomando conciencia cada vez más hoy en día, después que no sólo el actuar concreto de individuos y sociedades ha manifestado tal relativismo, sino que ha habido importantes esfuerzos en la cultura y el pensamiento modernos para hacerlo también teóricamente: disociando la política de la ética; proclamando la amoralidad de la creación cultural; afirmando la neutralidad ética de la ciencia y la tecnología; estableciendo la economía en lo cuantitativo e ignorando lo cualitativo de los valores; "sospechando" de las normas éticas y morales como "moralina", como sometidas al poder socio-económico o como represoras de impulsos vitales; finalmente, proclamando su relatividad, como productos históricos.

\section{— ¿Universalidad?}

Por otro lado, bien sabemos que el absolutismo valorativo puede conduciry ha conducido a dogmatismo, intolerancia y autoritarismo. De modo que la construcción de un universalismo axiológico no dogmático es un problema abierto.

Parece evidente que la mayor preocupación sobre la posibilidad de universalizar valores se da en el campo ético (que incluye el civico, según la manera aristotélica de considerar ética y política como ciencias "de fines").

En la actualidad, hay problemas planetarios y universales, cuya dimensión y respuestas éticas hay que descubrir y hacer reales. Tales problemas son los de la pobreza, la desigualdad cada vez más acentuada, las carencias materiales, de educación y la seguridad que sufren grandes masas humanas $y_{\text {, }}$ por otra parte, la acumulación fabulosa de riquezas por personas y grupos poderosos, o criminales. Instituciones políticas y sociales cada vez más desvirtuadas, reducidas a poco más que las declaraciones vacías de sus principios fundadores; derechos 
humanos cada vez menos respetados en los hechos; los recursos naturales cada vez más escasos y codiciados; la vida del planeta amenazada; un mundo de economía globalizada y de incomprensiones, conflictos e intolerancias cada vez más agudos.

Por otra parte, se hacen cada vez más perceptibles exigencias como: una relación equitativa y armónica entre los seres humanos y de unidad y armonía de cada ser humano en sí mismo; el amor y el respeto hacia la vida en todas sus manifestaciones, o, al menos, un uso reflexivo de los recursos del planeta; el que las organizaciones políticas y sociales remocen su ser y quehacer de acuerdo con su sentido y con sus responsabilidades; una economía armonizada con la ética y una tecnología al servicio de la humanidad y no sencillamente del poder; finalmente, un desarrollo humano ético, equitativo y sostenible, que implique la liberación del miedo y de la necesidad ( según el concepto de "seguridad humana" hecho propio por las $\mathrm{Na}$ ciones Unidas).

Indudablemente, la Filosofía posee una doble misión: la crítica y la propuesta de sabiduría. Sin olvidar la primera, que es garantía de lucidez, creemos que, hoy, una de las tareas más importantes y urgentes del filosofar es proponer concepciones y valores éticos y morales universales, a saber, no basados en un sistema doctrinario específico, sino en nociones y principios no excluyentes y comprensibles universalmente.

¿Existirá una base segura, aunque frágil, de una nueva estimativa, o ciencia de los valores que sea universal? Podría tal vez ser el Humanismo, este criterio antiguo y siempre nuevo para afirmar y proponer valores y para educar en ellos a las nuevas generaciones. Nacemos humanos, pero no basta, ya que hay que depurar y desarrollar la humanidad. Sin embargo, el concepto de humanismo se presta para ser relativizado. En efecto, con respecto al humanismo, las definiciones y las técnicas no nos quitan del todo la inquietud que nos provoca la diferencia entre predicar el humanismo, conocer el humanismo y practicar el humanismo. Incluso, no es lo mismo conocer y practicar el humanismo que creer conocerlo y practicarlo, con consecuencias, a veces, antihumanistas.

Otros autores mencionan, como orientación básica para nuestro mundo del siglo XXI, los Derechos Humanos, a saber, el reconocimiento del hombre por el hombre, "sin otra trascendencia que la voluntad de mantener a nuestros contemporáneos en un cierto ideal de promoción de la dignidad de todo ser humano en su vida cotidiana..." (Dutuis: 1997: 182). Sin embargo: ¿Hay universalidad de criterios en el reconocimiento de los Derechos Humanos?

Hoy cabe una posición menos perpleja y más "orgánica" a propósito de la formulación de valores objetivamente deseables y de una ética universal. El mundo en que vivimos cambia muy rápidamente y la incógnita sobre los valores deseables para la sociedad actual se podría estar despejando ya, o en un futuro no lejano. Esta aldea global, con sus comunicaciones tan fáciles y efectivas, podría ya estar sugiriendo a la reflexión axiológica propuestas válidas y universalmente aceptables.

Situaciones complejas, como las graves responsabilidades de la tecnociencia y la situación ambiental han hecho que autores connotados hayan formulado o estén tratando de formular los principios de una ética universal.

Así pues, el filósofo Hans Jonas (1903-1993) exige una conciencia ética aplicada a la ciencia y a la tecnología y considera que el descomunal poder que en la actualidad el hombre ha alcanzado sobre la naturaleza exige un principio nuevo en cuanto a su sujeto y a su objeto: el de Responsabilidad.

En un sentido que involucra más a fondo lo epistemológico, lo antropológico y lo cosmológico, los llamados "paradigmas emergentes", están proponiendo valores para toda la humanidad.

Desde este terreno que, al tener como base una visión sistémica, presenta una perspectiva 
de integración, E. Morin, el autor de la Teoría de la Complejidad, considera necesario superar la crisis de la ética más allá del nihilismo y del moralismo. Propone que el pensamiento adquiera la conciencia de lo complejo, lo sistémico y lo global. Propone, a nivel individual, la autoconciencia y la lucidez y lo que podríamos llamar un sentido de "pertenencia": a la especie humana, a la sociedad; finalmente, una conciencia planetaria. Todo eso lleva a una "ética de religación" que significa: comprensión, solidaridad, compasión, respeto, inclusión, tolerancia, amor, responsabilidad y lleva a una regeneración ética de la ciencia y de la política.

Desde el paradigma holístico y hologramático y la "ecología profunda", se propone el sentido de pertenencia a la Tierra, a la "trama de la vida" y al Universo. Se trata de una visión de religación que incluye, además, el sentirse parte de la humanidad, la visión de la unidad cuerpoalma, la paz, el respeto a la dignidad humana; una ética de la vida y la solidaridad planetaria. De estas concepciones, surge también una nueva experiencia de lo Sagrado, no institucional ni dogmática, en donde conspiran la visión sistémica de la ciencia y la espiritualidad.

Por otra parte, la visión sistémica de las organizaciones humanas propone que éstas adquieran la inteligencia de "pensar en sistemas"; su responsabilidad social y ambiental, la unión de la ética con las actividades económicas y de servicio, nuevos conceptos de liderazgo, formas más participativas de convivencia, de democracia y de disponibilidad de los bienes.

Las orientaciones pedagógicas que simpatizan con el posmodernismo, o comparten la crítica a la modernidad, promueven, en oposición a una razón autoritaria y homogeneizadora, los valores de la tolerancia activa; el respeto a las diferencias (culturales, raciales, étnicas, religiosas, de género y de opción sexual) y, por consiguiente, la inclusividad.

Lo aquí enunciado no es todavía un sistema ordenado de valores, pero sí representa la emergencia (concepto, por cierto, inherente a la noción de los "sistemas vivos") de intuiciones, sensibilidades, orientaciones intelectuales, que sugieren un nuevo panorama cultural y axiológico.

Por su parte, los nuevos paradigmas aluden, en el campo intelectual, a una nueva manera de conocer: separada del racionalismo reductivista mecanicista, disciplinario y especializado y claman por un conocimiento "sistémico", "complejo", "holístico", de conjuntos y no parcelizado, inter-, multi- y trans-disciplinario adecuado (aunque hoy se crea que el conocer es más una interpretación y una contrucción de la realidad, que una adecuación a ella) a una realidad unitaria e integrada.

\section{- Carencias y problemas}

A propósito de la limitada eficacia del sistema educativo a la hora de producir los valores pregonados, sean ellos intelectuales, morales, vitales, estéticos o espirituales, se nos plantea la famosa pregunta pedagógica del huevo y de la gallina: ¿es que la sociedad no los tiene y entonces no los sabe inspirar al sistema educativo, o es que el sistema educativo no tiene la capacidad para forjarlos e introducirlos en la sociedad?

Se dice, y es cierto, que nuestro país carece de plan educativo a largo plazo; que la familia está en crisis; que el desarrollo humano ha desmejorado en las áreas de educación, salud y superación de la pobreza, en particular se asiste a que el deterioro de la educación secundaria no está resuelto, que la violencia aumenta, promovida, entre otras cosas, por el aumento de la desigualdad; que la droga y el alcohol atraen cada vez más a los jóvenes. Estos son afectados por el debilitamiento de la vida familiar, que el acoso económico y la incertidumbre del futuro contribuyen a exacerbar; afectados también por la pobreza espiritual y humana de la sociedad consumista; por la falta de modelos de vida valiosos y atractivos; por el impacto descontrolado de medios de comunicación y espectáculos; desilusionados por una perversa traición de las palabras, perpetrada, con frecuencia, por 
quienes aspiran al poder o lo ostentan; por la inquietud de no poderse integrar a la vida productiva o a los estudios que les interesan.

La sociedad contemporánea, tan avanzada en ciencia y tecnología, sigue siendo sumamente inculta en lo que se refiere a la convivencia humana, a su organización, a su experiencia. Ahora bien, gran parte de las teorías educativas actuales, y no solo las utópicas, sino las científicas también, nos proponen una renovación de la convivencia, con base en el reconocimiento recíproco de los derechos humanos, en la equidad, en la tolerancia, la cooperación, la solidaridad, la comprensión, la prevención y resolución pacífica de los conflictos; basado, lo anterior, en la idea que solo un compromiso con valores éticos es capaz de garantizar la sostenibilidad del planeta en sus recursos ambientales, económicos, socio-culturales y humanos.

\section{E El "Cómo"}

Es obvio que la responsabilidad de la educación no se dirige únicamente a los valores éticos, sino a muchos otros aspectos: a todo el ámbito del conocimiento, representado por las materias de estudio, que debería dirigirse hacia la formación del pensamiento y del sentido crítico; a la formación de sanos criterios y buenos hábitos para la salud y la educación física; a la formación de criterios sobre lo bello y lo armonioso; a la adquisición de buenas normas de convivencia y sensibilidad social; a la sensibilidad religiosa y a la coherencia entre lo que se cree y lo que se hace, etc. Sin embargo, nada de esto debe resultar en una simple imposición de criterios y conductas. Primero que nada, entonces, hay que formar la estimativa, o el aprecio hacia los valores, como tratándose de una forma o estructura de la conciencia.

\section{¿Cómo, entonces, educar en valores?}

Hoy para todo buscamos definiciones precisas y técnicas para hacer las cosas. Hay que reconocer que es característica de la educación y del sistema educativo costarricense la fe, más que en teorías, en técnicas y métodos educativos, que sean científicos, a saber, definibles, reproducibles y de resultados previsibles, cuya aplicación debería garantizar los resultados.

Todavía no se ha resuelto (ni tal vez se resolverá) la disyuntiva de si la educación es ciencia, o es arte, o es intuición y espíritu creador. Es muy probable que no se trate de una disyuntiva y que lo sensato sea pensar que es al mismo tiempo todas estas cosas, de modo, que es importante conocer ciencias humanas y sociales y practicar técnicas y métodos educativos, y, al mismo tiempo, favorecer la creatividad y la intuición de unos docentes ricos en valores humanos y en sabiduría.

No es esta la ocasión oportuna para tratar exhaustivamente de los diferentes enfoques y métodos contemporáneos que se han enunciado para la educación en valores. Sin embargo, podemos mencionar, entre las corrientes educativas actuales, el Constructivismo, que, con su base en la psicología genética piagetiana, es seguramente la más en boga, incluso basada en las investigaciones de Piaget y seguidores sobre la formación del criterio moral en el niño y su visión del proceso desde la heteronomía hacia la autonomía y desde el autoritarismo hacia el consenso social y la cooperación acerca de las normas.

Otras corrientes de base psicológica, como la teoría de la Inteligencia Emocional y la teoría de las Inteligencias Múltiples, nos proponen criterios científicos, además que para la adquisición y educación de lo que se ha dado en llamar "destrezas sociales", también en autoconocimiento y manejo de la parte emocional individual, con el fin de introducir una cultura de menor agresividad y mayor comprensión, de empatía, de aceptación recíproca, de prevención de los conflictos.

En particular, el reconocimiento de múltiples tipos de inteligencia induce a mayor tolerancia 
y respeto de las diferencias, a la realización personal y al aprovechamento social de los talentos individuales, dentro y fuera del sistema educativo. Obviamente, es difícil el separar, en estas tendencias, aspectos metodológicos y aspectos de enunciación de valores y tiene poco sentido hacerlo, incluso, porque los valores enunciados son congruentes con una ética universalizable $y$, también, con una cultura de paz.

Sugerencias filosóficas contemporáneas sobre cómo educar en valores nos vienen, también, del Personalismo Educativo, concepción que hunde sus raíces en una amplia tradición del pensamiento occidental, como la aristotélicoescolástica, representada por la enseñanza de las virtudes como hábitos y fundamenta$\mathrm{da}$, desde el punto de vista antropológico, en el concepto de persona, el más importante en la tradición antropológica del cristianismo católico. "Persona" implica intelecto, voluntad y libertad, de modo, que la educación personalizada es un proceso de perfeccionamiento individual y, al mismo tiempo, de socialización y convivencia, en donde la autonomía personal y la libertad responsable son finalidad y medio de la educación.

Como base para formar en valores se puede considerar, así mismo, una "ética de consenso" como la "ética discursiva" de Habermas y Apel, entendidos los valores como principios éticos basados en argumentaciones que pueden ganarles el reconocimiento de todos los afectados, a través de un diálogo celebrado en condiciones de simetría. En la ética discursiva y en su fundamentación racional, podemos reconocer, como lo hace Adela Cortina, principios valiosos como la libertad, o autonomía de los interlocutores del diálogo; la solidaridad y la igualdad, que es otra palabra para indicar la simetría en el diálogo.

Otra perspectiva emancipadora y que posee una base teórica común con la socio-crítica de la cual también Habermas procede, es la de Paulo Freire. Desde la pedagogía del oprimido a la de la esperanza, el "cómo" de la pedagogía está estrechamente ligado con los ejes axiológicos (de valores), además que con una visión utópica: la transformación política mediante la superación histórica de las contradicciones sociales. El diálogo freireano es, a la vez, instrumento en el proceso de la liberación, a través de la conciencia lúcida, y portador de valores humanos: es instrumento de relación horizontal entre semejantes y diferentes; es condición de una cultura humanizante; es una actitud y una praxis que repudia el autoritarismo, la arrogancia, la intolerancia; los fundamentalismos. En el diálogo, los seres humanos se encuentran para la tarea común del saber y actuar dirigidos a procesos de transformación de la realidad.

Queremos poner de relieve dos criterios muy importantes sobre el "cómo" de la formación en valores. El primero es el principio constructivista que la formación moral debe ir paralela con la formación de la inteligencia. Piaget y su continuación constructivista tienen razón en vincular la actividad intelectual con la libertad moral: puesto que la educación forma un todo indisociable, no es posible formar unas personalidades autónomas en el terreno moral si, por otro lado, se las coacciona desde el punto de vista intelectual, haciendo que tengan que aprender "por encargo", sin descubrir por sí mismas la verdad.

El otro criterio con el que coincidimos es que si los valores se vinculan con la formación integral de la persona, es obvio que su enseñanza debe ser "transversal" con respecto al curriculum e involucrar las diferentes materias de enseñanza y no ser simple parte de éste, como una materia más.

\section{A modo de conclusión}

Como decíamos, es probablemente correcto afirmar que la educación es, al mismo tiempo, filosofía, ciencia, arte, método, intuición y creación. La educación en valores refleja tal cuestión de manera peculiar, porque los valores tienen que ver con las grandes preguntas 
sobre el ser y el sentido de la vida. No podemos hacer un discurso aséptico sobre este tema, ni enfocado sólo al "cómo": los valores no admiten ser tratados como "objetos" indiferentes a nuestras experiencias intelectuales y emocionales más profundas. También le decimos "no" al dogmatismo fundamentalista, que aplasta la autonomía de la conciencia en donde se generan los valores. Tampoco es admisible convertirlos en puro discurso o declaración retórica de principios, que no se convierta en experiencia y testimonio concreto, a través de nuestras acciones y de nuestra manera de ser. Si alguna conciencia crítica tenemos que adquirir es de la esquizofrenia que padece nuestro medio social por la dualidad entre los valores declarados, de los que tanto acostumbramos complacernos, y las vivencias concretas. Traducida en términos educativos, la vivencia de los valores se convierte en el mejor de los métodos: educar con el ejemplo.

Es necesario educar en valores por medio de los valores mismos, que deben estar presentes y tangibles en el sistema educativo. De modo que hay que remozar los valores del sistema educativo: en los contenidos; en los métodos; en el ambiente físico; en las relaciones entre las personas; en la ética docente; en el cumplimiento de todos; en las relaciones de autoridad; en la preocupación verdadera por la salud, la felicidad y la formación de los alumnos; en la conciencia crítica acerca de la sociedad y la cultura de hoy en día, y en el cumplimiento real de principios, normas y deberes.

\section{REFERENCIAS}

K.O. Apel, K.O. (1991). Verdad y responsabilidad. Barcelona: Paidós.

L.Boff, L. (2003). La voz del arco iris. Madrid: Trotta.

Capra, F. (1994). Pertenecer al universo. Madrid: EDAF.

Capra, F. (1999). La trama de la vida. Barcelona: Anagrama.

Capra, F.(2002). Las conexiones ocultas. Implicaciones sociales, medioambientales, económicas y biológicas de una nueva visión del mundo. Barcelona: Anagrama.

Cortina, A. Ética discursiva y educación en valores. Consultado en abril de 2010, http.//www.caredu.files.wordpress.com.

Delors, J. (1997). La educación encierra un tesoro. Informe a la UNESCO de la Comisión Internacional sobre la educación para el siglo XXI, Correo de la UNESCO.

Dutuis, J.C.E. (1997). Mediación y conciliación. Buenos Aires: Abeledo.

Ferrater Mora, J. (1958). Diccionario de Filosofía. Buenos Aires: Sudamericana.

Freire, P. (1996). Pedagogía de la esperanza (2 ${ }^{\mathrm{a}}$ ed.). México: Siglo XXI.

García Hoz, V. (1998). Educación personalizada (7 ${ }^{a}$ ed.). Bogotá: Grupo Editor Quinto Centenario.

Gardner, H. (1994). Estructuras de la mente. La teoría de las inteligencias múltiples. México: Fondo de Cultura Económica.

Goleman, D. (1996). La inteligencia emocional. Buenos Aires: Vergara.

Gordillo, M.V. (1992). Desarrollo moral y educación. Pamplona: EUNSA.

Habermas, J. (1985). Conciencia moral y acción comunicativa. Barcelona: Península.

Habermas, J. (1999). La inclusión del otro. Barcelona: Paidós.

Havelock, R.G., Huberman, A.M. (1980). Innovación y problemas de la educación. Teoría y realidad en los países en desarrollo. UNESCO.

Jonas, H. (1995). El principio de responsabilidad. Barcelona: Herder.

Kamii, C. (2001). La autonomía como finalidad de la educación. En Documentos del I Curso Internacional Hacia una cultura de la comprensión. Fundación Omar Dengo. San José, 5-6 de setiembre 2001.

Morin, E. (1999). Los siete saberes necesarios a la educación del futuro. París: UNESCO. 
Morin, E. (2004). La Méthode. 6 Éthique. París: Éditions du Sevil.

Morin, E. (2004). Tierra patria. Buenos Aires: Nueva Visión.

Ortega, P., Mínguez, R., Gil, R. (1996). Valores y educación. Barcelona: Ariel.

Piaget, J. (1935). El juicio moral en el niño. Madrid: Francisco Beltrán.
Sartori, G. (1999). Homo videns. México: Taurus.

Savater, F. (1999). El valor de educar. Barcelona: Ariel.

Senge, P. (2002). Escuelas que aprenden. Bogotá: Norma.

Organización de los Estados Iberoamericanos para la educación, la ciencia y la cultura. (1998). Educación, valores y democracia. Madrid. 